\title{
NORMAL RULED SURFACES OF A SURFACE ALONG A CURVE IN EUCLIDEAN 3-SPACE $\mathbb{E}^{3}$
}

\author{
R. A. ABDEL-BAKY ${ }^{1,2, *}$ AND S. H. NAZRA ${ }^{3}$ \\ ${ }^{1}$ Deptartment of Mathematics, Sciences Faculty for Girls, Jeddah University, 21352 Jeddah, KSA \\ ${ }^{2}$ Department of Mathematics, Faculty of Science, Assiut University, 71516 Assiut, EGYPT \\ ${ }^{3}$ Department of Mathematics, Umm Al-Qura University, KSA \\ *Corresponding author: rbaky@live.com
}

\begin{abstract}
In this paper, we define a ruled surface normal to a surface along a curve on the surface. Then, we analyze the necessary and sufficient condition for that surface to be normal developable. Also, we solve the problem when the resulting developable surface is a cylinder, cone or tangent surface. Finally, we give some representative examples.
\end{abstract}

\section{INTRODUCTION}

In differential geometry, moving frames constitute an important tool while studying curves and surfaces. The most familiar moving frames are the Frenet-Serret frame along a space curve, and the Darboux frame along a surface curve. In Euclidean 3 -space $\mathbb{E}^{3}$, the Darboux frame is constructed by the velocity of the curve and the normal vector of the surface whereas the Frenet-Serret frame is constructed from the velocity and the acceleration of the curve. Expressing the derivatives of these moving frames in terms of the frames themselves includes some real valued functions. These functions are called the curvature and the torsion for

Received 2019-04-20; accepted 2019-05-16; published 2019-07-01.

2010 Mathematics Subject Classification. 53A04, 53A05, 53A17.

Key words and phrases. Darboux frame; normal ruled surface; orthogonal trajectory.

(C)2019 Authors retain the copyrights of their papers, and all open access articles are distributed under the terms of the Creative Commons Attribution License. 
the Frenet-Serret frame, and the normal curvature, the geodesic curvature, and the geodesic torsion for the Darboux frame (see for example [1-3]).

The study of surfaces with a common characteristic curve plays an important role in a diversity of applications. In practical applications, the concept of family of surfaces having a given geodesic curve was first introduced by Wang et. al. [4] The basic idea is to regard the wanted surface as an extension from the given characteristic curve, and represent it as a linear combination of the marching-scale functions $u(s, t)$, $v(s, t), w(s, t)$ and the three vector functions $\mathbf{t}(s), \mathbf{n}(s), \mathbf{b}(s)$, which are the unit tangent, the principal normal and the binormal vector of the curve respectively. With the given geodesic curve and isoparametric constraints, they derived the necessary and sufficient conditions for the correct parametric representation of the surface pencil. The extension to ruled and developable surfaces is also outlined. This principal has been used treated extensively in the works (see for example [5-9]).

Properties of ruled surfaces and their offset surfaces with Darboux frame are defined by Senturk and Yuce $[11,12]$. Then, the ruled surfaces characteristic properties which are related to the geodesic curvature, the normal curvature and the geodesic torsion are investigated. Moreover, some theorems about the integral invariants of the ruled surface and their offset surfaces with Darboux frame are given. However, the relevant work on surfaces through characteristic curve on a surface is rare. So, this led us to offer an approach for designing surfaces from a given curve on a surface. For this purpose, we consider a curve on a surface and a ruled surface to the surface along the curve. Such a ruled surface is called a normal ruled surface along the curve if it exists. We have a special direction in the Darboux frame at each point of the curve which is written as a linear combination of the moving Darboux frame. Then, the extension to developable surfaces are also outlined. Meanwhile, we solved the problem when the resulting developable surface is a cylinder, cone or tangent surface. Finally, we illustrate the convenience and efficiency of this approach by some representative examples.

\section{Preliminaries}

The ambient space is the Euclidean 3 -space $\mathbb{E}^{3}$, and for our work we have used [1-3] as general references. A ruled surface in $\mathbb{E}^{3}$ is a surface generated by a straight line $L$ moving along a curve $\alpha(s)$. The various positions of the generating lines are called the rulings of the surface. Such a surface, thus, has a parametrization in the ruled form:

$$
\mathbf{y}(s, v)=\alpha(s)+v \mathbf{x}(s), v \in \mathbb{R}
$$

where $\alpha(s)$ is called the base curve, and the line passing through $\alpha(s)$ that is parallel to $\mathbf{x}(s)$ is called the ruling of the surface at $\alpha(s)$. The surface $\mathbf{y}(s, v)$ is regular if $\mathbf{y}_{s} \times \mathbf{y}_{v} \neq \mathbf{0}$ for all points, where $\mathbf{y}_{s}$ and $\mathbf{y}_{v}$ are the partial derivatives of $\mathbf{y}(s, v)$ with respect to $s$ and $v$, respectively. If there exists a common perpendicular to two constructive rulings in the ruled surface, then the foot of the common perpendicular on the main 
rulings is called a central point. The locus of the central point is called striction curve. The parametrization of the striction curve on the ruled surface is given by

$$
\mathbf{C}(s)=\alpha(s)-\frac{<\alpha^{\prime}, \mathbf{x}^{\prime}>}{\left\|\mathbf{x}^{\prime}\right\|^{2}} \mathbf{x}(s)
$$

We call a point of the ruled surface singular if the partial derivatives $\mathbf{y}_{s}$ and $\mathbf{y}_{v}$ are linearly dependent. The distribution parameter for a ruled surface describes the winding speed of the tangent planes winding about the ruling. It can be determined by

$$
\mu(s)=\frac{\operatorname{det}\left(\alpha^{\prime}, \mathbf{x}, \mathbf{x}^{\prime}\right)}{\left\|\mathbf{x}^{\prime}\right\|^{2}},
$$

which only depends on s. It's well known that $\mathbf{y}(s, v)$ is a developable surface if and only if $\operatorname{det}\left(\alpha^{\prime}, \mathbf{x}, \mathbf{x}^{\prime}\right)=0$. The relation between the Gaussian curvature $K$ and the distribution parameter is given by the formula:

$$
K(s, v)=-\frac{\mu^{2}}{\left(\mu^{2}+v^{2}\right)^{2}}<0 .
$$

If the ruled surface satisfies $\mathbf{y}(s, v)=\mathbf{y}(s+2 \pi, v)$ for all $s \in I$, then the ruled surface is called closed. We shall denote the closed normal ruled surface by $y$-closed ruled surface. A curve which intersects perpendicularly each one of rulings is called an orthogonal trajectory of the ruled surface. It is calculated by

$$
<d \mathbf{y}, \mathbf{x}>=0
$$

Let $\alpha: I \subseteq \mathbb{R} \rightarrow \mathbb{E}^{3}$ be a unit speed curve; by $\kappa(s)$ and $\tau(s)$ we denote the natural curvature and torsion of $\alpha=\alpha(s)$, respectively. We assume $\alpha^{\prime \prime}(s) \neq 0$ for all $s \in[0, L]$, since this would give us a straight line. In this paper, $\alpha^{\prime}(s)$ denote the derivative of $\alpha$ with respect to arc length parameter $s$. For each point of $\alpha(s)$, the set $\{\mathbf{t}(s), \mathbf{n}(s), \mathbf{b}(s)\}$ is called the Serret-Frenet frame along $\alpha(s)$, where $\mathbf{t}(s)=\alpha^{\prime}(s)$ is the unit tangent, $\mathbf{n}(s)=\alpha^{\prime \prime}(s) /\left\|\alpha^{\prime \prime}(s)\right\|$ is the unit principal normal, and $\mathbf{b}(s)=\mathbf{t}(s) \times \mathbf{n}(s)$ is the unit binormal vector. The arc-length derivative of the Serret-Frenet frame is governed by the relations:

$$
\left(\begin{array}{l}
\mathbf{t}^{\prime}(s) \\
\mathbf{n}^{\prime}(s) \\
\mathbf{b}^{\prime}(s)
\end{array}\right)=\left(\begin{array}{lll}
0 & \kappa(s) & 0 \\
-\kappa(s) & 0 & \tau(s) \\
0 & -\tau(s) & 0
\end{array}\right)\left(\begin{array}{c}
\mathbf{t}(s) \\
\mathbf{n}(s) \\
\mathbf{b}(s)
\end{array}\right)
$$

Let $M$ be a regular surface, and $\alpha: I \subseteq \mathbb{R} \rightarrow M$ is a unit speed curve on $M$. On the surface, we have the Darboux frame $\left\{\alpha(s) ; \mathbf{e}_{1}, \mathbf{e}_{2}, \mathbf{e}_{3}\right\} ; \mathbf{e}_{1}(s)$ be the unit tangent vector to $\alpha(s), \mathbf{e}_{3}=\mathbf{e}_{3}(s)$ is the surface unit normal restricted to $\alpha$, and $\mathbf{e}_{2}=\mathbf{e}_{3} \times \mathbf{e}_{1}$ be the unit tangent to the surface $M$. Then, the rotation matrix between Serret-Frenet frame and Darboux frame is

$$
\left(\begin{array}{l}
\mathbf{t}(s) \\
\mathbf{n}(s) \\
\mathbf{b}(s)
\end{array}\right)=\left(\begin{array}{lll}
1 & 0 & 0 \\
0 & \cos \vartheta & \sin \vartheta \\
0 & -\sin \vartheta & \cos \vartheta
\end{array}\right)\left(\begin{array}{l}
\mathbf{e}_{1} \\
\mathbf{e}_{2} \\
\mathbf{e}_{3}
\end{array}\right)
$$


Then we have the following Darboux formulae:

$$
\left(\begin{array}{l}
\mathbf{e}_{1}^{\prime} \\
\mathbf{e}_{2}^{\prime} \\
\mathbf{e}_{3}^{\prime}
\end{array}\right)=\left(\begin{array}{lll}
0 & \kappa_{g} & \kappa_{n} \\
-\kappa_{g} & 0 & \tau_{g} \\
-\kappa_{n} & -\tau_{g} & 0
\end{array}\right)\left(\begin{array}{l}
\mathbf{e}_{1} \\
\mathbf{e}_{2} \\
\mathbf{e}_{3}
\end{array}\right)=\omega(s) \times\left(\begin{array}{l}
\mathbf{e}_{1} \\
\mathbf{e}_{2} \\
\mathbf{e}_{3}
\end{array}\right),
$$

where $\omega(s)=\tau_{g} \mathbf{e}_{1}-\kappa_{n} \mathbf{e}_{3}+\kappa_{g} \mathbf{e}_{3}$ is referred to as the Darboux vector. Here,

$$
\begin{aligned}
& \kappa_{n}(s)=\kappa \sin \vartheta=\kappa_{n}(u)=<\alpha^{\prime \prime}(s), \mathbf{e}_{3}> \\
& \kappa_{g}(s)=\kappa \cos \vartheta=\operatorname{det}\left(\alpha^{\prime}(s), \alpha^{\prime \prime}(s), \mathbf{e}_{3}(s)\right) \\
& \tau_{g}(s)=\tau-\vartheta^{\prime}=\operatorname{det}\left(\alpha^{\prime}(s), \mathbf{e}_{3}(s), \mathbf{e}_{3}^{\prime}(s)\right)
\end{aligned}
$$

We call $\kappa_{g}=\kappa_{g}(s)$ a geodesic curvature, $\kappa_{n}=\kappa_{n}(s)$ a normal curvature, and $\tau_{g}=\tau+\vartheta^{\prime}$ a geodesic torsion of $\alpha(s)$, respectively. In terms of these quantities, the geodesics, line of curvatures, and asymptotic lines on a smooth surface may be characterized, as loci along which $\kappa_{g}=0, \tau_{g}=0$, and $\kappa_{n}=0$, respectively.

The angle of pitch of a closed ruled surface

When the set $\left\{\alpha(s) ; \mathbf{e}_{1}(s), \mathbf{e}_{2}(s), \mathbf{e}_{3}(s)\right\}$ is transposed to the origin $\mathbf{0}$ by the translation $\alpha(s) \rightarrow \mathbf{0}$, it performs there a spherical motion about the fixed center $\mathbf{0}$. According to the elements of spherical kinematics the motion is an infinitesimal rotation about an instantaneous axis whose direction is given by the Darboux vector $\omega(s)$. The corresponding Steiner vector of the motion is

$$
\mathbf{S}(s)=\oint \omega=\left(\oint \tau_{g}\right) \mathbf{e}_{1}-\left(\oint \kappa_{n}\right) \mathbf{e}_{3}+\left(\oint \kappa_{g}\right) \mathbf{e}_{3}
$$

The angle of pitch of the $y$-closed ruled surface is defined by

$$
\lambda_{x}=<\mathbf{x}, \mathbf{S}>
$$

Thus the Steiner vector will be

$$
\mathbf{S}(s)=\lambda_{1} \mathbf{e}_{1}-\lambda_{2} \mathbf{e}_{2}+\lambda_{3} \mathbf{e}_{3},
$$

where $\lambda_{i}=\lambda_{i}(s)(\mathrm{i}=1,2,3)$ are the angle of pitches of the ruled surfaces

$$
\mathbf{y}_{i}(s, v)=\alpha(s)+v \mathbf{e}_{i}(s), v \in \mathbb{R} .
$$

2.1. Normal ruled surfaces. We now design normal ruled surfaces along the curve $\alpha(s)$ of $M$ as follows: A straight line $L$ in $\mathbb{E}^{3}$ such that it is strictly connected to Darboux frame of the unit speed curve $\alpha(s)$ of the given surface $M$ is represented, uniquely with respect to this frame, in the form

$$
\left.\begin{array}{l}
\mathbf{x}(s)=x_{1}(s) \mathbf{e}_{1}(s)+x_{2}(s) \mathbf{e}_{2}(s)+x_{3}(s) \mathbf{e}_{3}(s), \\
x_{1}^{2}+x_{2}^{2}+x_{3}^{2}=1, \mathbf{x}^{\prime} \neq \mathbf{0}
\end{array}\right\}
$$


where the components $x_{i}=x_{i}(s)(\mathrm{i}=1,2,3)$ are scalar functions of the arc length parameter $s$ of the base curve $\alpha(s)$.

Let the set $\left\{\mathbf{e}_{1}, \mathbf{e}_{2}, \mathbf{e}_{3}\right\}$ complete a closed motion along the base curve $\alpha(s) \in M$. Then the straight line $L$ generates a closed ruled surface. We can describe the surface by the equation

$$
M_{n}: \mathbf{P}(s, v)=\alpha(s)+v \mathbf{x}(s), v \in \mathbb{R}
$$

To solve our constrained design problem, the normal vector at the point $\mathbf{P}(s, 0)$ is

$$
\left(\mathbf{P}_{s} \times \mathbf{P}_{v}\right)(s, 0)=x_{2} \mathbf{e}_{3}-x_{3} \mathbf{e}_{2}
$$

Now, it seems natural to pose the following question. Under what condition $M_{n}$ is normal ruled surface of the surface $M$ ?. The answer is affirmative and can be stated as follows: $M_{n}$ is normal ruled surface of the surface $M$ if and only if $x_{3} \neq 0$ and $x_{2}=0$. By substitution:

$$
x_{1}(s)=<\mathbf{e}, \mathbf{e}_{1}>=\cos \varphi, \quad x_{3}(s)=<\mathbf{e}, \mathbf{e}_{3}>=\sin \varphi \neq 0,
$$

so that we have

$$
\left.\begin{array}{l}
M_{n}: \mathbf{P}(s, v)=\alpha(s)+v \mathbf{x}(s), v \in \mathbb{R}, \\
\mathbf{x}=\cos \varphi \mathbf{e}_{1}+\sin \varphi \mathbf{e}_{3} .
\end{array}\right\}
$$

When we choose different $\varphi_{0}$, we can get different surfaces. We can control the shape of the surfaces by the value of $\varphi(s)$. We can also calculate that the unit normal vector to the normal ruled surface $M_{n}$ at $\mathbf{P}(s, v)$ is:

$$
\mathbf{u}(s, v):=\frac{\mathbf{P}_{s} \times \mathbf{P}_{v}}{\left\|\mathbf{P}_{s} \times \mathbf{P}_{v}\right\|}=-\frac{\mu \mathbf{e}_{2}+v \mathbf{e}_{2} \times \mathbf{x}}{\sqrt{\mu^{2}+v^{2}}},
$$

which is the normal $\mathbf{u}_{0}$ at the point $\mathbf{P}(s, 0)$. Let $\phi$ be the angel between the unit normal vectors $\mathbf{u}$ and $\mathbf{u}_{0}$, then

$$
\tan \phi=\frac{v}{\mu} .
$$

This result is an expression of the well known Chasles Theorem: When $\mu>0(\mu<0)$ the tangent plane turns counterclockwise (clockwise) and the ruled surface is called left-handed (right-handed) ruled surface. As an immediate result we state that: The tangent plane turns evidently through $\pi$ along a ruling in a non-developable $(\mu \neq 0)$ normal ruled surface $M_{n}$.

According to Eqs. (2.2), and (2.17) the striction curve is given by:

$$
\left.\begin{array}{l}
\mathbf{C}(s)=\alpha(s)-\sigma(s) \mathbf{x}(s), \\
\text { where } \sigma(s)=\frac{\left(\kappa_{n}+\varphi^{\prime}\right) \sin \varphi}{\left(\kappa_{n}+\varphi^{\prime}\right)^{2}+\left(\kappa_{g} \cos \varphi-\tau_{g} \sin \varphi\right)^{2}} .
\end{array}\right\}
$$


Also, the distribution parameter is

$$
\mu(s)=\frac{\left(\tau_{g} \sin \varphi-\kappa_{g} \cos \varphi\right) \sin \varphi}{\left(\kappa_{n}+\varphi^{\prime}\right)^{2}+\left(\kappa_{g} \cos \varphi-\tau_{g} \sin \varphi\right)^{2}} .
$$

Via Eqs. (2.4), and (2.21), the Gaussian curvature is

$$
\left.\begin{array}{l}
K(s, v)=-\frac{\left(\tau_{g} \sin \varphi-\kappa_{g} \cos \varphi\right)^{2} \sin ^{2} \varphi}{\left[1-2 v\left(\kappa_{g}+\varphi^{\prime}\right) \sin \varphi+\cos \varphi+v^{2} f(s)\right]^{2}}, \\
\text { where } f(s)=\left[\left(\kappa_{g} \cos \varphi-\tau_{g} \sin \varphi\right)^{2}+\left(\kappa_{n}+\varphi^{\prime}\right)^{2} \cos ^{2} \varphi\right] .
\end{array}\right\}
$$

Theorem 1. For the $M_{n}$-closed ruled surface of $M$ along $\alpha(s)$, the Gaussian curvature $K(s, v)$ along a ruling takes the maximum value at the striction point on that ruling.

Proof. According to Eq. (2.22), we have:

$$
\frac{\partial K(s, v)}{\partial v}=-\frac{\partial}{\partial v}\left[\frac{\left(\tau_{g} \sin \varphi-\kappa_{g} \cos \varphi\right)^{2} \sin ^{2} \varphi}{\left[1-2 v\left(\kappa_{g}+\varphi^{\prime}\right) \sin \varphi+\cos \varphi+v^{2} f(s)\right]}\right],
$$

from which we have

$$
\frac{\partial K(s, v)}{\partial v}=0 \Leftrightarrow v=\frac{\left(\kappa_{n}+\varphi^{\prime}\right) \sin \varphi}{\left(\kappa_{n}+\varphi^{\prime}\right)^{2}+\left(\kappa_{g} \cos \varphi-\tau_{g} \sin \varphi\right)^{2}}=\sigma(s) .
$$

Thus, $v$ gives us the maximum of $K(s, v)$ since

$$
\left.\frac{\partial^{2} K(s, v)}{\partial v^{2}}\right|_{v}<0
$$

Thus $K(s, v)$ has its maximum value at the central point on each of the rulings since the central point corresponds to the value $v=\sigma(s)$. This completes the proof of the theorem.

The pitch of a closed ruled surface

Consider $v=v(s)$ as a function of $s$ with continuous derivatives of a certain order such that the regular curve:

$$
\Gamma: \beta(s)=\alpha(s)+v(s) \mathbf{x}(s)
$$

on the $M_{n}$-closed ruled surface of $M$ along $\alpha(s)$ is orthogonal to the generator, then we have

$$
<\beta^{\prime}, \mathbf{x}>=0 \Rightarrow<\alpha^{\prime}, \mathbf{x}>+v^{\prime}=0 .
$$

The pitch of the $M_{n}$-closed ruled surface is

$$
L(s)=\oint<\beta^{\prime}, \mathbf{x}>d s=-\oint d v=v_{0}-v_{1} .
$$

This equation shows that, in general, $\Gamma$ is not a closed curve. After a period, curve $\Gamma$, starting from the point $p_{0}$ on the generator intersects the same generator at another point $p_{1}$ which is generally different from 
$p_{0}$, i.e. $L_{i}=p_{0} p_{1}$. Eq. (2.25) can be also expressed as an integral invariant of the $M_{n}$-closed ruled surface: By means of Eqs. (2.8), and (2.24) we may write

$$
\beta^{\prime}=\left[1-v\left(\kappa_{n}+\varphi^{\prime}\right) \sin \varphi\right] \mathbf{e}_{1}+v\left[\left(\kappa_{g} \cos \varphi-\tau_{g} \sin \varphi\right) \mathbf{e}_{2}+\left(\kappa_{n}+\varphi^{\prime}\right) \cos \varphi \mathbf{e}_{3}\right]
$$

and therefore Eq. (2.25) becomes

$$
L(s)=\oint \cos \varphi d s
$$

which is the desired result. Also, it can be shown that the tangle of pitch is given by

$$
\lambda(s)=\lambda_{1} \cos \varphi+\lambda_{3} \sin \varphi
$$

Corollary 1. For the $M_{n}$-closed ruled surface of $M$ along $\alpha(s)$, the short distance between two rulings is the distance measured only on the striction curve which is one of the orthogonal trajectories.

Proof. Fixing two rulings, say for $s_{1}<s_{2}$, we compute the length $\eta(v)$ of an orthogonal trajectory between these two rulings by

$$
\eta(v)=\int_{s_{1}}^{s_{2}}\left\|\beta^{\prime}\right\| d s=\sqrt{\left[1-v\left(\kappa_{n}+\varphi^{\prime}\right) \sin \varphi\right]^{2}+v^{2} f^{2}} d s
$$

where

$$
f(s)=\left[\left(\kappa_{g} \cos \varphi-\tau_{g} \sin \varphi\right)^{2}+\left(\kappa_{n}+\varphi^{\prime}\right)^{2} \cos ^{2} \varphi\right]
$$

To find value of $v$ which minimizes $\eta(v)$, we have to use $\frac{\partial \eta(v)}{\partial v}=0$ which gives $v=\sigma(s)$. This completes the proof.

Example 1. Suppose we are given a parametric curve

$$
\alpha(u)=\left(1+\cos u, \sin u, 2 \sin \frac{u}{2}\right)
$$

After simple computation, we have

$$
\mathbf{e}_{1}(u)=\frac{\dot{\alpha}(u)}{\|\dot{\alpha}(u)\|}=\left(-\frac{\sqrt{2} \sin u}{\sqrt{3+\cos u}}, \frac{\sqrt{2} \cos u}{\sqrt{3+\cos u}}, \frac{\sqrt{2} \cos \frac{u}{2}}{\sqrt{3+\cos u}}\right) .
$$

Then

$$
M: \mathbf{X}(u, v)=\left(1+\cos u-\frac{\sqrt{2} v \sin u}{\sqrt{3+\cos u}}, \frac{\sqrt{2} v \cos u}{\sqrt{3+\cos u}}+\sin u, \frac{\sqrt{2} v \cos u}{\sqrt{3+\cos u}}+2 \sin \frac{u}{2}\right)
$$

which is known as the tangent surface of $\alpha(u)$. It follows that:

$$
\begin{aligned}
\mathbf{e}_{3}(u) & =\frac{\mathbf{X}_{u} \times \mathbf{X}_{v}}{\left\|\mathbf{X}_{u} \times X_{v}\right\|} \\
& =\left(\frac{-3 \sin \frac{u}{2}-\sin \frac{3 u}{2}}{\sqrt{13+3 \cos u}}, \frac{2 \sqrt{2} \cos \left(\frac{u}{2}\right)^{3}}{\sqrt{13+3 \cos u}}, \frac{-2 \sqrt{2}}{\sqrt{13+3 \cos u}}\right) .
\end{aligned}
$$


Since $\ddot{\alpha}(u)=\left(-\cos u,-\sin u,-\frac{1}{2} \sin \frac{u}{2}\right)$, we have:

$$
\begin{aligned}
\kappa_{g}(u) & =\frac{\operatorname{det}\left(\dot{\alpha}(u), \ddot{\alpha}(u), \mathbf{e}_{3}(u)\right)}{\|\dot{\alpha}(u)\|^{3}}=-\sqrt{\frac{13+3 \cos u}{(3+\cos u)^{3}}}, \\
\kappa_{n}(u) & =\frac{<\ddot{\alpha}(u), \mathbf{e}_{3}(u)>}{\|\dot{\alpha}(u)\|^{2}}=0, \\
\tau_{g}(u) & =\frac{\operatorname{det}\left(\dot{\alpha}(u), \mathbf{e}_{3}(u), \dot{\mathbf{e}}_{3}(u)\right)}{\|\dot{\alpha}(u)\|^{2}}=\frac{6 \cos \frac{u}{2}}{13+3 \cos u},
\end{aligned}
$$

so that $\alpha(u)$ is an asymptotic on $M$. Then we have a normal ruled surface family given by

$$
M_{n}: \mathbf{P}(u, v)=\left(1+\cos u, \sin u, 2 \sin \frac{u}{2}\right)+v\left(\cos \varphi \mathbf{e}_{1}+\sin \varphi \mathbf{e}_{3}\right), v \in \mathbb{R}
$$

If we take $\varphi(u)=u$, then we immediately obtain a member of this family given by

$$
M_{n}: \mathbf{P}(u, v)=(u \cos u, u \sin u, 5 u)+v\left(\cos u \mathbf{e}_{1}+\sin u \mathbf{e}_{3}\right), v \in \mathbb{R} .
$$

The striction curve is

$$
\begin{aligned}
\mathbf{C}(u)= & \alpha(u)+\frac{\sin u}{\left(\kappa_{n}+1\right)} \mathbf{x}(u) \\
= & \left\{\begin{array}{c}
1+\cos (u)+\sin (u)\left(-\frac{\sqrt{2} \cos (u) \sin (u)}{\sqrt{3+\cos (u)}}-\frac{\sin (u)\left(3 \sin \left(\frac{u}{2}\right)+\sin \left(\frac{3 u}{2}\right)\right)}{\sqrt{26+6 \cos (u)}}\right) \\
\sin (u)+\sqrt{2} \sin (u)\left(\frac{\cos (u)^{2}}{\sqrt{3+\cos (u)}}+\frac{2 \cos \left(\frac{u}{2}\right)^{3} \sin (u)}{\sqrt{13+3 \cos (u)}}\right) \\
2 \sin \left(\frac{u}{2}\right)+\sqrt{2} \sin (u)\left(\frac{\cos \left(\frac{u}{2}\right) \cos (u)}{\sqrt{3+\cos (u)}}-\frac{2 \sin (u)}{\sqrt{13+3 \cos (u)}}\right)
\end{array}\right\},
\end{aligned}
$$

So that, for the curvature functions of $M_{n}$ we have

$$
\begin{aligned}
\mu(u)= & -\frac{\sin (u)\left(\frac{\cos (u) \sqrt{13+3 \cos (u)}}{(3+\cos (u))^{3 / 2}}+\frac{6 \cos \left(\frac{u}{2}\right) \sin (u)}{13+3 \cos (u)}\right)^{2}}{1+\left(\frac{\cos (u) \sqrt{13+3 \cos (u)}}{(3+\cos (u))^{3 / 2}}+\frac{6 \cos \left(\frac{u}{2}\right) \sin (u)}{13+3 \cos (u)}\right)^{2}}, \\
K(u, v)= & -\frac{\sin (u)^{2}\left(\frac{\cos (u) \sqrt{13+3 \cos (u)}}{(3+\cos (u))^{3 / 2}}+\frac{6 \cos \left(\frac{u}{2}\right) \sin (u)}{13+3 \cos (u)}\right)^{2}}{\left(1+\cos (u)-2 v \sin (u)+v^{2}\left(f(u)^{2}\right)\right)^{2}}, \sin (u)=(-4.7) \sin (u), \\
\lambda(u)= & \left(\int_{0}^{2 \pi}-\frac{\sqrt{13+3 \cos (u)}}{(3+\cos (u))^{3 / 2}} d u\right) \\
L(s)= & \int_{0}^{2 \pi} \cos u d u=0 .
\end{aligned}
$$

where

$$
f(u)=\cos (u)^{2}+\left(\frac{\cos (u) \sqrt{13+3 \cos (u)}}{(3+\cos (u))^{3 / 2}}+\frac{6 \cos \left(\frac{u}{2}\right) \sin (u)}{13+3 \cos (u)}\right)^{2}
$$



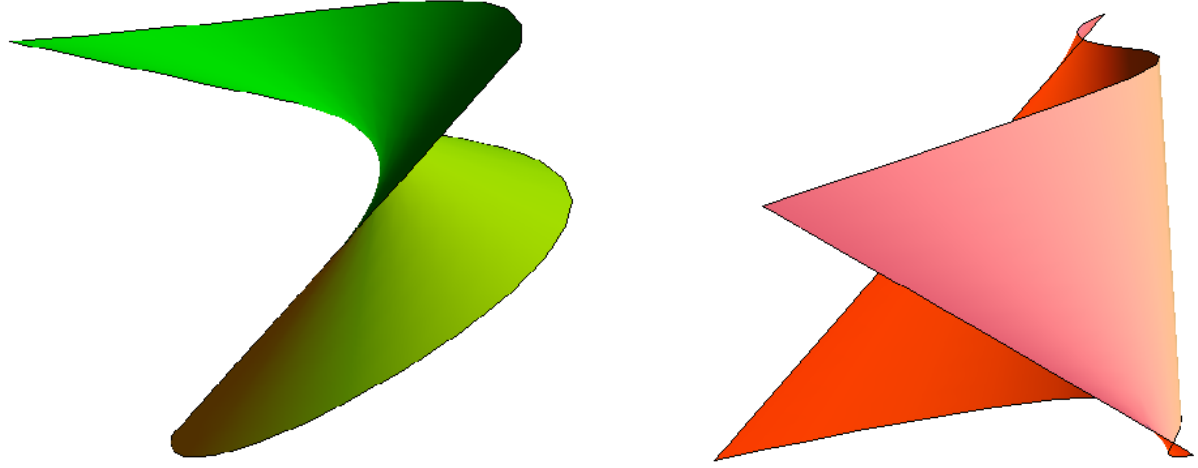

Figure 1. The surface $M$

Figure 2. The $M_{n}$ ruled surface

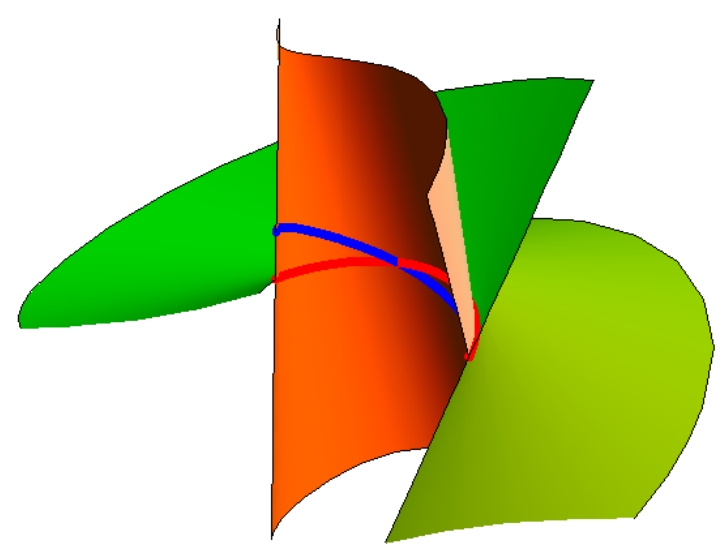

Figure 3. $M_{n} \cup M$ with the base curve (red) $\alpha(u)$ and the striction curve (blue) $C(u)$

2.2. Normal developable ruled surface. A developable surface is a special ruled surface which has the same tangent planes along a generator, or to which the tangent planes along a ruling coincide. In view of Eq. (2.3), we have:

$$
\mu(s)=\operatorname{det}\left(\alpha^{\prime}, \mathbf{x}, \mathbf{x}^{\prime}\right)=0 \Leftrightarrow\left(\kappa_{g} \cos \varphi-\tau_{g} \sin \varphi\right) \sin \varphi=0 .
$$

We will now investigate this condition in detail: if $\sin \varphi=0$, then $M_{n}$ can not be normal developable surface (In fact we have imposed $\varphi \neq 0$ ). Then, according to the assumption of $M_{n}$ being a normal developable ruled surface $\left(M_{n}\right.$-developable for short) of $M$, we have $\kappa_{g} \cos \varphi-\tau_{g} \sin \varphi=0$, and consequently from Eq. $(2.17)$ :

$$
\left.\begin{array}{l}
M_{n}: \mathbf{P}(s, v)=\alpha(s)+v \mathbf{x}(s), v \in \mathbb{R}, \\
\mathbf{x}(s)=\cos \varphi \mathbf{e}_{1}+\sin \varphi \mathbf{e}_{3}, \\
\cos \varphi=\frac{\tau_{g}}{\sqrt{\tau_{g}^{2}+\kappa_{g}^{2}}}, \text { and } \sin \varphi=\frac{\kappa_{g}}{\sqrt{\tau_{g}^{2}+\kappa_{g}^{2}}} \neq 0 .
\end{array}\right\}
$$


Theorem 2. The necessary and sufficient condition for $M_{n}$-developable being a normal along $\alpha(s)$ of $M$ is that there exist a parameter $v \in \mathbb{R}$ and a function $\varphi(s)=\tan ^{-1} \frac{\kappa_{g}}{\tau_{g}}$, so that $M_{n}$ can be represented by Eq. $(2.29)$.

As is customary in the literature, there are three types of developable surfaces, the given curve can be classified into three kinds correspondingly. In what follows, we will discuss the relationship between the given curve $\alpha(s) \in M$ and its isoparametric developable. In the light of Eq. (2.28), we have:

$$
<\mathbf{x} \times \mathbf{x}^{\prime}, \alpha^{\prime}>=0
$$

The first case is when,

$$
\mathbf{x} \times \mathbf{x}^{\prime}=\mathbf{0} \Leftrightarrow\left(\kappa_{n}+\varphi^{\prime}\right) \mathbf{e}_{2}=\mathbf{0}
$$

In this case, $M_{n}$ is referred to as a cylindrical surface. Since $\mathbf{e}_{2}$ is a nonzero unit vector, then the $M_{n^{-}}$ developable is a cylindrical surface if and only if

$$
\varphi(s)=\varphi_{0}-\int_{s_{0}}^{s} \kappa_{n} d s,
$$

where $\varphi_{0}=\varphi\left(s_{0}\right)$, and $s_{0}$ is the starting value of the arc length.

By similar argument, we can also have the following:

$$
\mathbf{x} \times \mathbf{x}^{\prime} \neq \mathbf{0}
$$

This implies that the $M_{n}$-developable is a non-cylindrical surface. Therefore, the first derivative of the directrix is

$$
\alpha^{\prime}(s)=\mathbf{C}^{\prime}(s)+\sigma(s) \mathbf{x}^{\prime}(s)+\sigma^{\prime}(s) \mathbf{x}(s)
$$

where $\mathbf{C}^{\prime}$ is the first derivative of the striction curve, $\sigma(s)$ is a smooth function. Substituting Eq. (2.34) into Eq. (2.30) gives:

$$
<\mathbf{x} \times \mathbf{x}^{\prime}, \mathbf{C}^{\prime}>=0
$$

Similarly, there are two possible cases which satisfy Eq. (2.35), as presented in the following: The first case is when the first derivative of the striction curve is $\mathbf{C}^{\prime}=0$. Geometrically this condition implies that the striction curve degenerates to a point, and the ruled surface becomes a cone; the striction point of a cone is commonly referred to as the vertex. Therefore, the $M_{n}$-developable is a cone if and only if there exists a fixed point $\mathbf{C}$ and a function $\sigma(s)$ such that:

$$
\left.\begin{array}{l}
1+\sigma\left(\kappa_{n}+\varphi^{\prime}\right) \sin \varphi-\sigma^{\prime} \cos \varphi=0, \\
\sigma\left(\kappa_{n}+\varphi^{\prime}\right) \cos \varphi+\sigma^{\prime} \sin \varphi=0,
\end{array}\right\}
$$


which imply

$$
\sigma(s)=-\frac{\sin \varphi}{\kappa_{n}+\varphi^{\prime}} .
$$

The second case is when $\mathbf{C}^{\prime} \neq 0$, i.e. $\sigma(s) \neq-\frac{\sin \varphi}{\kappa_{n}+\varphi^{\prime}}$. From Eq. (2.35), $\mathbf{C}^{\prime}$ is perpendicular to $\mathbf{x} \times \mathbf{x}^{\prime}$, and, therefore, $\mathbf{C}^{\prime}$ is in the plane generated by $\mathbf{x}$ and $\mathbf{x}^{\prime}$. The condition for $\mathbf{C}$ to be striction curve is therefore equivalent to $\mathbf{C}^{\prime}$ and $\mathbf{x}^{\prime}$ are perpendicular to each other. Therefore, we may conclude that the ruling is parallel to the first derivative of the striction curve, which is also the tangent of the striction curve. This ruled surface is referred too as a tangent ruled surface. So, the $M_{n}$-developable represented by Eq. (2.29) is a tangent surface if and only if there exists a curve $\mathbf{C}(s)$ so that:

$$
\sigma(s) \neq-\frac{\sin \varphi}{\kappa_{n}+\varphi^{\prime}}
$$

So that, for the $M_{n}$-developable, we have:

$$
\left.\begin{array}{l}
\mathbf{C}(s)=\alpha(s)+\frac{\sin \varphi}{\kappa_{n}+\varphi^{\prime}} \mathbf{x}(s), \\
\lambda(s)=\lambda_{1} \cos \varphi+\lambda_{3} \sin \varphi, \\
L(s)=\oint \frac{\tau_{g}}{\sqrt{\tau_{g}^{2}+\kappa_{g}^{2}}} d s .
\end{array}\right\}
$$

Theorem 3 (Existence and uniqueness). Under the above notations there exists a unique $M_{n}$-developable ruled represented by Eq. (2.29).

Proof. For the existence, we have the $M_{n}$-developable along $\alpha=\alpha(s)$ represented by Eq. (2.29). On the other hand, since $M_{n}$ is a ruled surface, we assume that

$$
\begin{aligned}
& M_{n}: \mathbf{P}(s, v)=\alpha(s)+v \zeta(s), v \in \mathbb{R}, \text { with }\left(\tau_{g}, \kappa_{n}\right) \neq(0,0) \\
& \zeta(s)=\zeta_{1}(s) \mathbf{e}_{1}+\zeta_{2}(s) \mathbf{e}_{2}+\zeta_{3}(s) \mathbf{e}_{3} \\
& \|\zeta(s)\|^{2}=\zeta_{1}^{2}+\zeta_{2}^{2}+\zeta_{3}^{2}=1, \zeta^{\prime}(s) \neq \mathbf{0}
\end{aligned}
$$

It can be immediately seen from Eqs. (2.8) and (2.38) that $M_{n}$-ruled is developable if and only if

$$
\operatorname{det}\left(\alpha^{\prime}, \zeta, \zeta^{\prime}\right)=0 \Leftrightarrow-\zeta_{3} \zeta_{2}^{\prime}+\zeta_{2} \zeta_{3}^{\prime}-\zeta_{1}\left(\zeta_{3} \kappa_{g}-\zeta_{2} \kappa_{n}\right)+\tau_{g}\left(\zeta_{2}^{2}+\zeta_{3}^{2}\right)=0
$$

On the other hand, since $M_{n}$ is a developable surface which is normal developable surface along $\alpha=\alpha(s)$, we have

$$
\left(\mathbf{P}_{s} \times \mathbf{P}_{v}\right)(s, v)=\psi(s, v) \mathbf{e}_{2},
$$

where $\psi=\psi(s, v)$ is a differentiable function. Moreover, the normal vector $\mathbf{P}_{s} \times \mathbf{P}_{v}$ at the point $(s, 0)$ is

$$
\left(\mathbf{P}_{s} \times \mathbf{P}_{v}\right)(s, 0)=-\zeta_{3} \mathbf{e}_{2}+\zeta_{2} \mathbf{e}_{3}
$$


Thus, from Eqs. (2.39) and (2.40), one finds that:

$$
\zeta_{2}=0, \text { and } \zeta_{3}=-\psi(s, 0)
$$

which follows from Eq. (2.38) that

$$
\begin{gathered}
-\zeta_{1}\left(\zeta_{3} \kappa_{g}\right)+\tau_{g}\left(\zeta_{3}^{2}\right)=0 \\
\zeta_{3}\left(-\zeta_{1} \kappa_{g}+\zeta_{3} \tau_{g}\right)=0 .
\end{gathered}
$$

If $(s, 0)$ is a regular point (i.e., $\psi(s, 0) \neq 0)$, then $\zeta_{3}(s) \neq 0$. Thus, we have

$$
\zeta_{1}=\frac{\tau_{g}}{\kappa_{g}} \zeta_{3}, \text { with } \kappa_{g} \neq 0
$$

Therefore, we obtain

$$
\zeta(s)=\frac{\tau_{g}}{\kappa_{g}} \zeta_{3} \mathbf{e}_{1}+\zeta_{3} \mathbf{e}_{3}=\frac{\zeta_{3}}{\sin \varphi} \mathbf{e}(s), \text { with } \varphi \neq 0 .
$$

This means that the direction of $\zeta(s)$ is equal to the direction of $\mathbf{e}(s)$. If $\tau_{g} \neq 0$ (i.e., $\varphi \neq \frac{\pi}{2}$ ), we have the same result as the above case. On the other hand, suppose that $M_{n}$-developable has a singular point at $\left(s_{0}, 0\right)$. Then $\psi\left(s_{0}, 0\right)=\zeta_{2}\left(s_{0}\right)=\zeta_{3}\left(s_{0}\right)=0$, and we have $\zeta\left(s_{0}\right)=\zeta_{1}\left(s_{0}\right) \mathbf{e}_{1}\left(s_{0}\right)$. If the singular point $\alpha\left(s_{0}\right)$ is in the closure of the set of points where the $M_{n}$-developable along $\alpha(s)$ is regular, then there exists a point $\alpha(s)$ in any neighborhood of $\alpha\left(s_{0}\right)$ such that the uniqueness of the $M_{n}$-developable holds at $\alpha(s)$. Passing to the limit $s \rightarrow s_{0}$, uniqueness of the normal developable at $s_{0}$. Suppose that there exists an open interval $J \subseteq I$ such that $M_{n}$ is singular at $\alpha(s)$ for any $s \in J$. Then $\mathbf{P}(s, v)=\alpha(s)+v \zeta_{1}(s) \mathbf{e}_{1}(s)$ for any $s \in J$. This means that $\zeta_{2}(s)=\zeta_{3}(s)=0$ for $s \in J$. It follows that

$$
\left(\mathbf{P}_{s} \times \mathbf{P}_{v}\right)(s, v)=v \zeta_{1}^{2}\left(\kappa_{n} \mathbf{e}_{2}-\kappa_{g} \mathbf{e}_{3}\right)
$$

Thus the above vector is directed to $\mathbf{e}_{2}$, i.e. $\mathbf{P}_{s} \times \mathbf{P}_{v} \| \mathbf{e}_{3}(s)$ if and only if $\kappa_{n} \neq 0$ and $\kappa_{g}=0$ for any $s \in J$. In this case, $\mathbf{x}(s)= \pm \mathbf{e}_{3}$. This means that uniqueness holds. This ends the proof.

Example 2. By making use of Eqs. (2.29), and Example 1, the corresponding surfaces are shown in Figures 4,5 and 6 . 


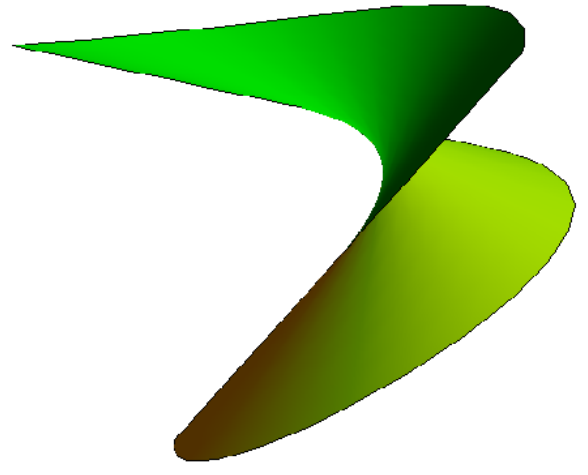

Figure 4 . The surface $M$

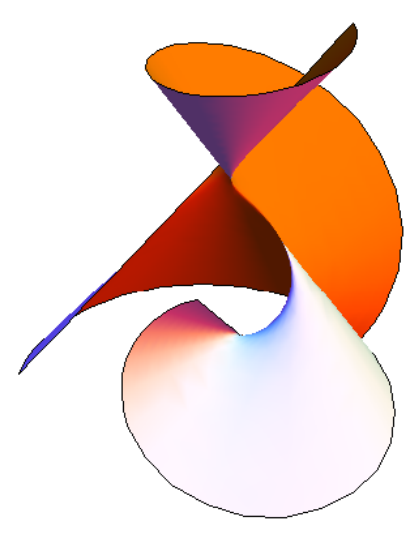

Figure 5. The $M_{n}$ developable surface

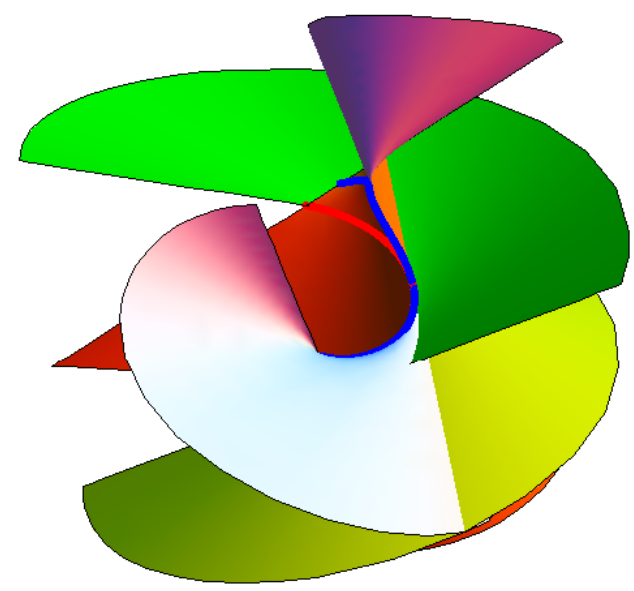

Figure 6. $M_{n} \bigcup M$ with the base curve (red) $\alpha(u)$ and the striction curve (blue) $C(u)$

On the other hand, we have assumed that $\tau_{g}^{2}+\kappa_{g}^{2} \neq 0$. If $\kappa_{g}=0$, and $\tau_{g}=0$, then $L_{x}(s)$ is undefined, and we have the following theorem:

Theorem 4. Let $\alpha: I \subseteq \mathbb{R} \rightarrow M$ be a unit speed curve on with $\kappa_{g}(s)=\tau_{g}(s)=0$. Then, the $M_{n^{-}}$ developable of $M$ along $\alpha(s)$ is a plane if and only if $\alpha$ is a ruling of $M_{n}$.

Proof. In general, the torsion of the curve as a space curve is given by

$$
\tau(s)=\tau_{g}+\frac{\kappa_{g} \kappa_{n}^{\prime}-\kappa_{n} \kappa_{g}^{\prime}}{\kappa_{g}^{2}+\kappa_{n}^{2}} .
$$

If $\kappa_{g}(s)=\tau_{g}(s)=0$, then $\tau=0$, so that $\alpha$ is a plane curve. Moreover, we have $\mathbf{e}_{2}^{\prime}=\mathbf{0}$. Thus, the $M_{n}$-developable is a plane along $\alpha$ of $M$. Since $\alpha$ is the intersection of $M$ with the plane $M_{n}, \alpha$ is a ruling.

Conversely, if $\alpha$ is a ruling of $M_{n}$-developable, then there exists a plane $\pi$ such that $\alpha(I)=M_{n} \cap M$, and $\mathbf{e}_{1}, \mathbf{e}_{3} \in \pi$ for any $s \in I$. Therefore, $\pi$ is orthogonal to $\mathbf{e}_{2} \in \pi$ for any $s \in I$. Since $\pi$ is a plane, $\pi$ is a $M_{n}$-developable along $\alpha \in M_{n}$. This ends the proof. 
As a result the following corollary can be given.

Corollary 2. Let $\alpha: I \subseteq \mathbb{R} \rightarrow M$ be a unit speed curve on with $\tau_{g}^{2}+\kappa_{g}^{2} \neq 0$. If there are two $M_{n^{-}}$ developable surfaces along $\alpha$, then $\alpha$ is a straight line.

Proof. Under the assumption of $\tau_{g}^{2}+\kappa_{g}^{2} \neq 0$ the $M_{n}$-ruled developable along $\alpha \in M_{n}$ is unique by Theorem 3. If there are two $M_{n}$-developable surfaces along $\alpha$ with $\kappa_{g}(s)=\tau_{g}(s)=0$, then $\alpha$ is a ruling for these two $M_{n}$-developable surfaces. If $\kappa_{g}(s)=\tau_{g}(s)=0$ at a point $s_{0}$ in the closure of the set of points where $\tau_{g}^{2}+\kappa_{g}^{2} \neq 0$, then there exists a point $s$ in any neighborhood of $s_{0}$ such that the uniqueness of the $M_{n^{-}}$ developable surface holds at $s$. Passing to the limit $s \rightarrow s_{0}$; the uniqueness of the $M_{n}$-developable surface holds at $s_{0}$. This completes the proof.

2.3. Special curves on a surface. In this subsection we consider special curves on a surface.

Firstly, we consider geodesics on surfaces. Let $\alpha: I \subseteq \mathbb{R} \rightarrow M$ be a unit speed curve. Since $\alpha$ is a geodesic of $M$, we have $\kappa_{g}=0$, and therefore from Eq. (2.9) $\kappa_{n}= \pm \kappa$, and $\tau_{g}=\tau$. If we replace $\kappa_{n}, \tau_{g}$, and $\kappa_{g}$ in Eq. (2.29), we have

$$
M_{n}: \mathbf{P}(s, v)=\alpha(s)+v \mathbf{e}_{1}(s), v \in \mathbb{R},
$$

which is known as the tangent developable surface of $\alpha(s)$. Also, in view of Eqs. (2.37), we get:

$$
\mathbf{C}(s)=\alpha(s), \lambda(s)=\lambda_{1} \text {, and } L(s)=\oint d s .
$$

In this case $L(s)$ equals the length of the striction curve of $M_{n}$, and conversely, if $L(s)$ is equal to the striction curve of $M_{n}$, then $M_{n}$-developable is a tangential developable. Hence the following Corollary is true:

Corollary 3. The $M_{n}$-developable is a tangential developable if and only if its pitch $L$ is equal to the length of the striction curve.

Example 3. Let $M$ be given as the following parameterization:

$$
M: \mathbf{X}(s, v)=\left(\frac{1}{\sqrt{2}} \cos s, \frac{1}{\sqrt{2}} \sin s, \frac{s}{\sqrt{2}}-2 v\right) .
$$

The Darboux frame which belong to this surface are found as:

$$
\begin{aligned}
& \mathbf{e}_{1}(s)=\left(-\frac{1}{\sqrt{2}} \sin \frac{s}{\sqrt{2}}, \frac{1}{\sqrt{2}} \cos \frac{s}{\sqrt{2}}, \frac{1}{\sqrt{2}}\right), \\
& \mathbf{e}_{2}(s)=\left(-\frac{1}{\sqrt{2}} \sin \frac{s}{\sqrt{2}}, \frac{1}{\sqrt{2}} \cos \frac{s}{\sqrt{2}},-\frac{1}{\sqrt{2}}\right), \\
& \mathbf{e}_{3}(s)=(-\cos s,-\sin s, 0)
\end{aligned}
$$

Moreover, we have

$$
\kappa_{g}(s)=0, \kappa_{n}(s)=\frac{1}{\sqrt{2}}, \text { and } \tau_{g}(s)=\frac{1}{\sqrt{2}}
$$


Thus, the $M_{n}$ - tangential developable is

$$
M_{n}: \mathbf{P}(s, v)=\left(\frac{1}{\sqrt{2}} \cos s, \frac{1}{\sqrt{2}} \sin s, \frac{s}{\sqrt{2}}\right)+v \cos \left(\frac{1}{\sqrt{2}}\right) \mathbf{e}_{1}(s)
$$

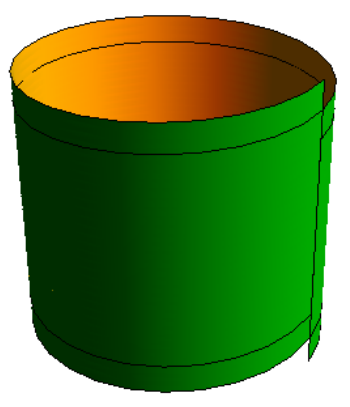

FIgURE 7 . The surface $M$

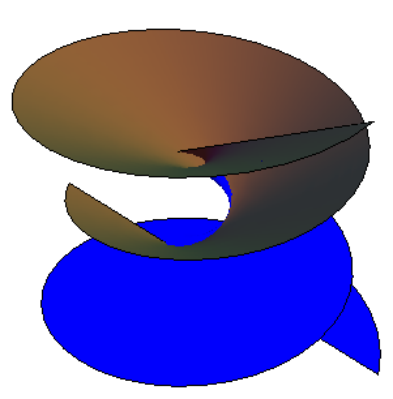

Figure 8. The $M_{n}$ tangential surface

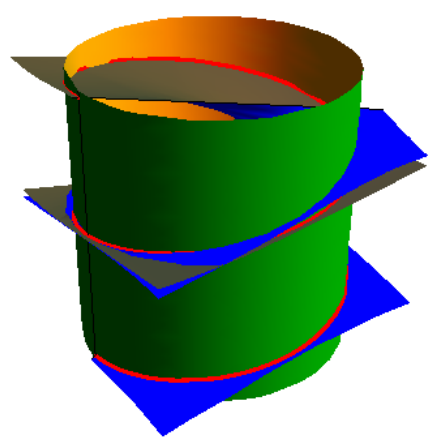

FiguRE 9. $M_{n} \bigcup M$ with the base curve $\alpha(s)$.

Secondly, we consider lines of curvature. Let $\alpha: I \subseteq \mathbb{R} \rightarrow M$ be a unit speed curve line of curvature with $\kappa_{g} \neq 0$. Since $\alpha$ is a line of curvature, we have $\tau_{g}=0$, and consequently from Eqs. (2.29) and (2.37) we obtain

$$
M_{n}: \mathbf{P}(s, v)=\alpha(s)+v \mathbf{e}_{3}(s), v \in \mathbb{R},
$$

so that

$$
\mathbf{C}(s)=\alpha(s)+\frac{1}{\kappa_{n}} \mathbf{e}_{3}(s), \lambda(s)=\lambda_{3}, \text { and } L(s)=0 .
$$

As an application of Eqs. (2.31) and (2.34) we conclude that:

(1)- $M_{n}$ is a circular cone if and only if $\kappa_{n}$ is constant (see Figures 10, 11, 12).

(2)- $M_{n}$ is a cylindrical surface if and only if $\kappa_{n}=0$ (see Figures $13,14,15$ ).

$$
\lambda(s)=\sqrt{2} \pi, \text { and } L(s)=\sqrt{2} \pi .
$$


Example 4. Let $M$ be given as the following parameterization:

$$
M: \mathbf{X}(s, v)=\left(\cos s-\frac{v}{\sqrt{2}} \cos s, \sin s-\frac{v}{\sqrt{2}} \sin s, \frac{v}{\sqrt{2}}\right) .
$$

The Darboux frame which belong to this surface are found as:

$$
\left.\begin{array}{l}
\mathbf{e}_{1}(s)=(-\sin s, \cos s, 0), \\
\mathbf{e}_{2}(s)=\left(-\frac{1}{\sqrt{2}} \cos s,-\frac{1}{\sqrt{2}} \sin s, \frac{1}{\sqrt{2}}\right), \\
\mathbf{e}_{3}(s)=\left(\frac{1}{\sqrt{2}} \cos s, \frac{1}{\sqrt{2}} \sin s, \frac{1}{\sqrt{2}}\right)
\end{array}\right\}
$$

Also, we have:

$$
\kappa_{g}(s)=\frac{1}{\sqrt{2}}, \kappa_{n}(s)=\frac{-1}{\sqrt{2}}, \text { and } \tau_{g}(s)=0
$$

The $M_{n}$-cone is

$$
M_{n}: \mathbf{P}(s, v)=(\cos s, \sin s, 0)+v \sin \left(\frac{1}{\sqrt{2}}\right) \mathbf{e}_{3}(s)
$$

By Eqs. (2.36), we find

$$
\lambda(s)=\sqrt{2} \pi, \text { and } L(s)=0
$$

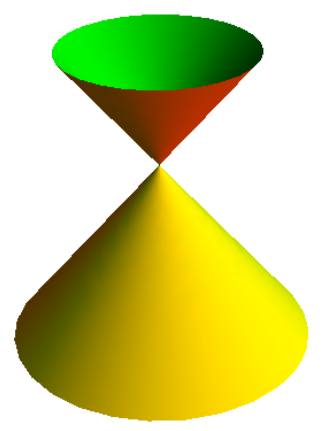

Figure 10. The surface $M$.

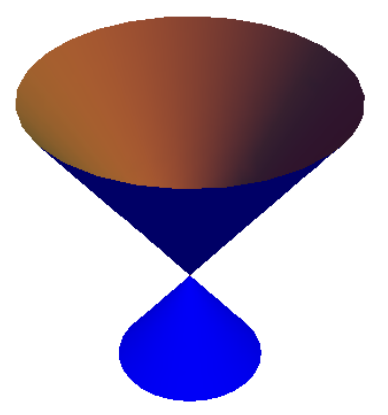

Figure 11. The $M_{n}$ cone.

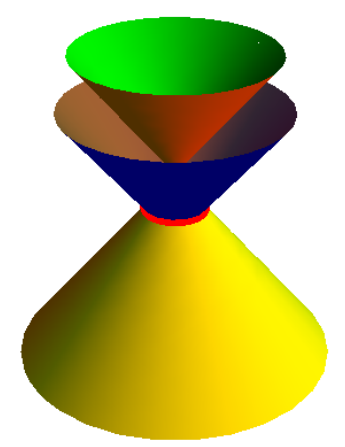

FiguRE 12. $M_{n} \bigcup M$ with the base curve $\alpha(s)$.

Thirdly, we consider asymptotic curve. Let $\alpha: I \subseteq \mathbb{R} \rightarrow M$ be a unit speed asymptotic curve with $\tau_{g}^{2}+\kappa_{g}^{2} \neq 0$. Since $\alpha$ is an asymptotic curve, we have $\kappa_{n}=0$, and consequently from Eqs. (2.7), (2.8), (2.29) 
and (2.37), we have:

$$
\left.\begin{array}{l}
M_{n}: \mathbf{P}(s, v)=\alpha(s)+v \mathbf{x}(s), v \in \mathbb{R}, \\
\mathbf{x}(s)=\cos \varphi \mathbf{t}+\sin \varphi \mathbf{b}, \\
\cos \varphi=\frac{\tau}{\sqrt{\tau^{2}+\kappa^{2}}}, \text { and } \sin \varphi=\frac{\kappa}{\sqrt{\tau^{2}+\kappa^{2}}} \neq 0,
\end{array}\right\}
$$

and

$$
\left.\begin{array}{l}
\mathbf{C}(s)=\alpha(s)+\frac{\sin \varphi}{\varphi^{\prime}} \mathbf{x}(s), \\
\lambda(s)=\lambda_{1} \cos \varphi+\lambda_{3} \sin \varphi, \\
L(s)=\oint \frac{\tau}{\sqrt{\tau^{2}+\kappa^{2}}} d s .
\end{array}\right\}
$$

Then the following corollary can be derived.

Corollary 4. The necessary and sufficient condition for the $M_{n}$-developable surface of $M$ along $\alpha(s)$ being as an asymptotic is that there exist a function $\varphi(s)=\cot ^{-1} \frac{\tau}{\kappa}$, so that $M_{n}$ can be represented by Eqs. (2.46).

Corollary 4 . shows that the property of the constructed $M_{n}$-developable is completely determined by the given asymptotic, and so is the type of the surface. Since there are three types of $M_{n}$-developable surfaces, the given curve can be classified into three kinds correspondingly. In what follows, we will discuss the relationship between the given asymptotic and its isoparametric $M_{n}$-developable of $M$ along $\alpha(s)$.

Suppose the surface constructed by Eq. (2.46) is a cylinder, then there is $\mathbf{x}^{\prime} \times \mathbf{x}=\mathbf{0}$, which results in $\kappa \tau^{\prime}-\tau \kappa^{\prime}=0$, namely $\left(\frac{\tau}{\kappa}\right)^{\prime}=0$. The ratio of curvature to torsion is a constant, and so the curve $\alpha(s) \in M$ is a generalized helix. Hence, we can state the following.

Corollary 5. The $M_{n}$-developable with $\alpha(s)$ is an asymptotic is a cylinder surface if and only if $\alpha(s)$ is a generalized helix.

Example 5. We consider asymptotic curve: $\alpha(s)=\left(\frac{4}{5} \cos s, 1-\sin s, \frac{-3}{5} \cos s\right)$, and let $M$ be given as the following parameterization:

$$
M: \mathbf{X}(s, v)=\left(\frac{4}{5} \cos s-\frac{4}{5} v-\frac{3}{5} v^{2}, 1-\sin s, \frac{-3}{5} \cos s+\frac{3}{5} v-\frac{4}{5} v^{2}\right)
$$

After straightforward calculations, we have

$$
\kappa_{g}(s)=\frac{\sqrt{2} \cos s}{\sqrt{1+\cos 2 s}}, \kappa_{n}(s)=0, \text { and } \tau_{g}(s)=0
$$


The $M_{n}$-cylinder is given by

$$
M_{n}: \mathbf{P}(s, v)=\left\{\begin{array}{c}
\frac{1}{5} \cos (s)\left(4+v\left(-4+\frac{\sqrt{2}(-3+8 v)}{\sqrt{1+8 v^{2}+\cos (2 s)}}\right) \sin (s)\right), \\
1-v \cos ^{2}(s)-\sin (s)-\frac{2 \sqrt{2} v^{2} \sin ^{2}(s)}{\sqrt{1+8 v^{2}+\cos (2 s)}}, \\
\frac{1}{5} \cos (s)\left(-3+v\left(3-\frac{2 \sqrt{2}(2+3 v)}{\sqrt{1+8 v^{2}+\cos (2 s)}}\right) \sin (s)\right)
\end{array}\right\}
$$
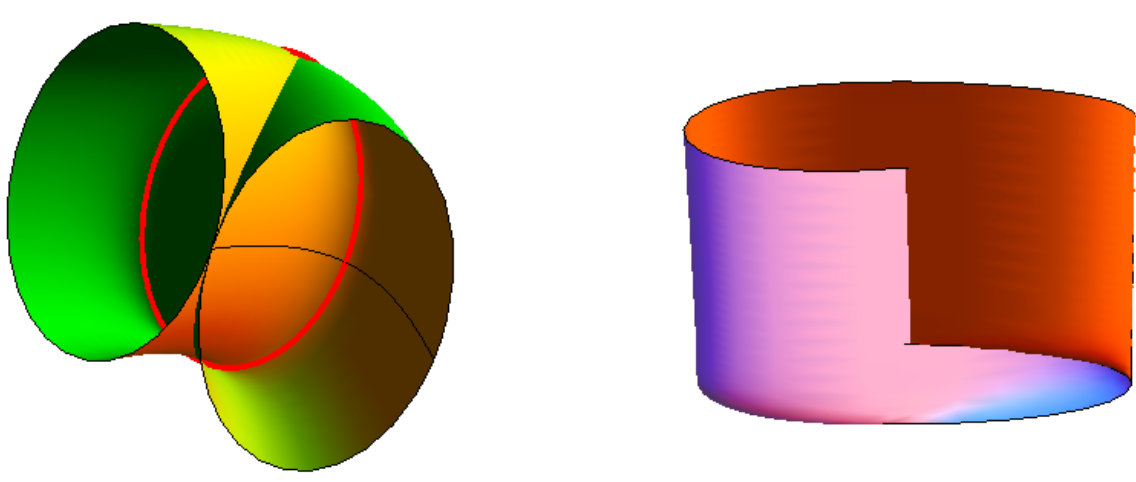

FiguRe 13. The surface $M$

Figure 14. The surface $M_{n}$

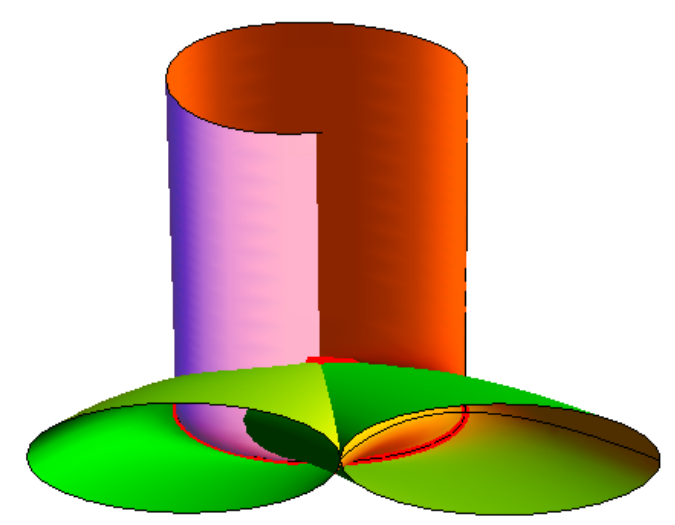

Figure 15. $M_{n} \bigcup M$ with the base curve $\alpha(s)$.

Suppose the $M_{n}$ developable is a cone. Then, as mentioned above, from Eqs. (2.6) and (2.46), we have:

$$
\mathbf{C}^{\prime}(s)=\left(1-\sigma^{\prime} \cos \varphi+\sigma \varphi^{\prime} \sin \varphi\right) \mathbf{t}-\left(\sigma^{\prime} \sin \varphi+\sigma \varphi^{\prime} \cos \varphi\right) \mathbf{b},
$$

which follows that $\mathbf{P}(s, u)$ is a circular cone if and only if

$$
\mathbf{0}=\left(1-\sigma^{\prime} \cos \varphi+\sigma \varphi^{\prime} \sin \varphi\right) \mathbf{t}-\left(\sigma^{\prime} \sin \varphi+\sigma \varphi^{\prime} \cos \varphi\right) \mathbf{b} .
$$


We have found, by equating the coefficients of $\mathbf{t}$ and $\mathbf{b}$, that:

$$
\left.\begin{array}{l}
1-\sigma^{\prime} \cos \varphi+\sigma \varphi^{\prime} \sin \varphi=0 \Leftrightarrow 1-\frac{d}{d s}(\sigma \cos \varphi)=0 \Leftrightarrow \sigma \cos \varphi=s+c_{1}, \\
\sigma^{\prime} \sin \varphi+\sigma \varphi^{\prime} \cos \varphi=0 \Leftrightarrow \frac{d}{d s}(\sigma \sin \varphi)=0 \Leftrightarrow \sigma \sin \varphi=c_{2} .
\end{array}\right\}
$$

By simple computation, we have $\frac{\tau}{\kappa}(s)-\frac{\tau}{\kappa}(0)=\frac{s}{c}$, where $c$ is an arbitrary constant.

Corollary 6. Let $M_{n}$ be the normal developable with $\alpha(s)$ expressed by Eq. (2.46). Then we have the following:

(A) $-M_{n}$ is a cone if and only if $\frac{\tau}{\kappa}(s)-\frac{\tau}{\kappa}(0)=\frac{s}{c}$,

(B)- $M_{n}$ is a tangential developable surface if and only if $\frac{\tau}{\kappa}(s)-\frac{\tau}{\kappa}(0) \neq \frac{s}{c}$.

\section{Conclusion}

In this paper, we study the problem of finding a family of normal ruled surfaces from a given curve on surface. We obtain the parametric representation for a ruled surface family whose members share the same curve as an isoparametric curve. Using the Darboux frame of the given curve on surface, we present the ruled surface as a linear combination of this frame and analyze the necessary and sufficient condition for that surface to be normal ruled surface. The extension to developable surfaces is also outlined. We illustrate this method by presenting some examples. Hopefully these results will lead to a wider usage of surfaces in geometric modeling, garment-manufacture industry, and the manufacturing of products.

\section{REFERENCES}

[1] B. O’Neill. Elementary Differential Geometry. Academic Press Inc., New York 1966.

[2] M.P. Do Carmo. Differential Geometry of Curves and Surfaces. Englewood Cliffs: Prentice Hall, 1976.

[3] G. Farin. Curves and Surfaces for Computer Aided Geometric Design, 2nd ed. New York: Academic Press, 1990.

[4] G.J. Wang, K. Tang, and C.L. Tai. Parametric representation of a surface pencil with a common spatial geodesic, Comput.Aided Des., 36 (2004), 447-459.

[5] E. Kasap, F.T. Akyildiz, K. Orbay. A generalization of surfaces family with common spatial geodesic, Appl. Math. Comput., 201 (2008), 781-789.

[6] G. Saffak, E. Kasap. Family of surface with a common null geodesic, Internat. J. Phys. Sci., 4(8) (2009), $428-433$.

[7] C.Y. Li, R.H. Wang, C.G. Zhu. Parametric representation of a surface pencil with a common line of curvature, Comput. Aided Des., 43 (9) (2011), 1110-1117.

[8] E. Bayram, F. Guler, E. Kasap. Parametric representation of a surface pencil with a common asymptotic curve, Comput. Aided Des., 44 (2012), 637-643.

[9] C.Y. Li, R.H. Wang, C.G. Zhu. An approach for designing a developable surface through a given line of curvature, Comput. Aided Des., 45 (2013) 621-627.

[10] G.Y. Senturk, S. Yuce. Characteristic properties of the ruled surface with Darboux frame. Kuwait J. Sci. 42 (2) (2015), $14-33$.

[11] G.Y. Senturk, S. Yuce. Bertrand offsets of ruled surfaces with Darboux Frame, Result. Math. 72 (3) (2017), 1151-1159. 